\title{
Asymptotic laws for knot diagrams
}

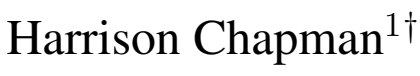 \\ ${ }^{1}$ University of Georgia, Athens, GA
}

\begin{abstract}
We study random knotting by considering knot and link diagrams as decorated, (rooted) topological maps on spheres and sampling them with the counting measure on from sets of a fixed number of vertices $n$. We prove that random rooted knot diagrams are highly composite and hence almost surely knotted (this is the analogue of the Frisch-Wasserman-Delbrück conjecture) and extend this to unrooted knot diagrams by showing that almost all knot diagrams are asymmetric. The model is similar to one of Dunfield, et al.

Résumé. Nous étudions les noeuds aléatoires en considérant les diagrammes de noeuds comme cartes planaires décorées sur lesquelles nous mettons la mesure uniforme de l'espace des cartes de $n$ sommets ( $n$ fixé). Nous prouvons que les diagrammes de noeuds enracinés aléatoires sont très "composés" et de fait presque sûrement noués (ceci est un équivalent de la conjecture de Frisch-Wasserman-Delbrück). Nous étendons ensuite ce résultat aux diagrammes de noeuds non enracinés en montrant que tous les diagrammes de noeuds sont asymétriques. Ce modèle est similaire à celui de Dunfield, et al.
\end{abstract}

Keywords. knots, maps, enumeration, immersed curves, DNA topology

\section{Introduction}

A planar map (or simply map) is a graph embedded in the sphere $S^{2}$ up to continuous deformation. It is 4-valent if the underlying graph is (i.e. all vertices are of degree 4); we will call 4-valent maps link shadows. Vertices of link shadows will be called crossings. A map is decorated by some set if there is a mapping from the vertices of the map to the set. A link component of a link shadow is an equivalence class of edges meeting opposite across crossings. If a link shadow consists of precisely one link component, it is called a knot shadow. We note that knot shadows are interesting in their own right; they are precisely curve immersions on the sphere studied by Gauss, Arnol'd (1995), and others.

The motivation for these definitions is thus: A knot type is an embedding of the circle $S^{1}$ into the 3 -sphere $S^{3}$ modulo "ambient isotopy." Call a knot (resp. link) shadow decorated with $\{+,-\}$ a knot (link) diagram; this sign information is represented graphically as "over-under" information (Figure 2 . Equivalently then, a knot type is an equivalence class of knot diagrams modulo the Reidemeister moves (essentially moves one can do with string, c.f. Figure 3). The unknot is the unique knot type represented by the trivial loop in $S^{3}$. The unknot is trivial, and diagrams representing another knot type are knotted.

The study of random knotting arises in numerous areas, principal among which is polymer physics: Polymers (such as DNA or proteins) are considered to be strings in space and in many cases their function

\footnotetext{
†Email: hchapmanemath.uga.edu

1365-8050 (c) 2016 Discrete Mathematics and Theoretical Computer Science (DMTCS), Nancy, France
} 

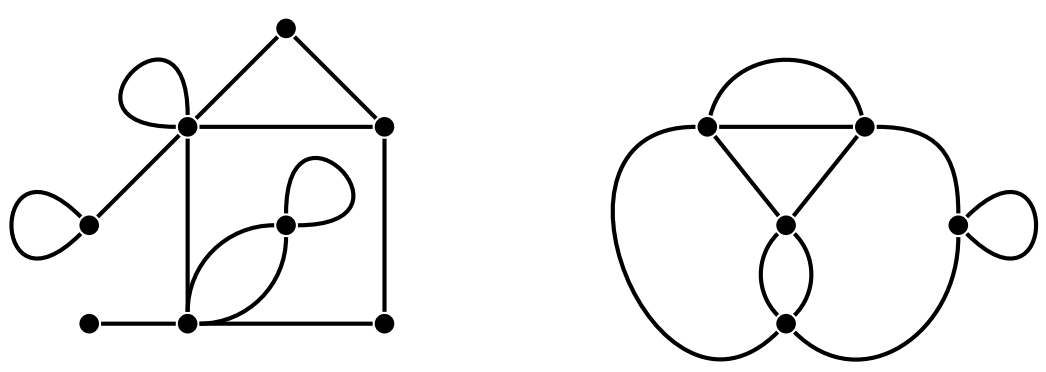

Fig. 1: A planar map (left) and a planar map in the class of knot shadows (right).

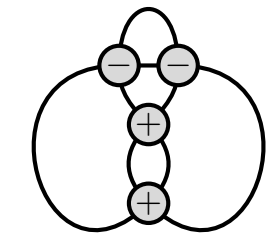

(a) A figure-eight diagram viewed as a decorated shadow.

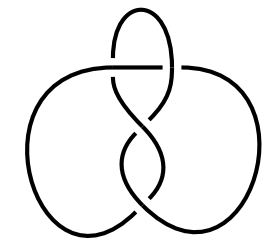

(b) A figure-eight diagram viewed as a knot drawing.

Fig. 2: After choosing once and for all a way to view signs as "over-under" information (i.e. orientation around the knot), knot diagrams can be drawn as usual.
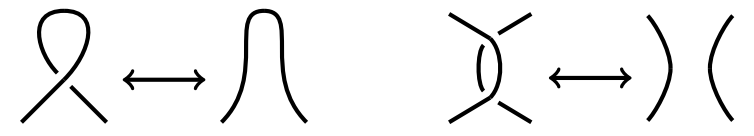

$\frac{Y}{\prime} \stackrel{\frac{1}{1}}{1}$

Fig. 3: The three Reidemeister moves. 
(or lack thereof) depends on any "knots" that appear within. The random diagram model of random knotting is then: Given a number $n$ of crossings, sample uniformly an unlabeled knot diagram with $n$ crossings and return its knot type. It is similar to models of Diao et al. (2012) and Dunfield et al. (2014), but these models do not sample from any well-understood measure on spaces of knot diagrams.

In the context of DNA topology, Frisch and Wasserman (1961) and Delbrück (1962) independently conjectured;

Conjecture (Frisch-Wasserman-Delbrück) As the size $n$ of a randomly sampled knot grows large, the probability that it is knotted tends to 1.

The first proof of the conjecture was for $n$-step self-avoiding lattice polygons, by Sumners and Whittington (1988):

Theorem (Sumners-Whittington) As the number of steps $n$ of a self-avoiding lattice polygon grows large, the probability that the polygon is knotted tends to 1 exponentially quickly.

Shortly thereafter the conjecture was proved in view of other models of space curves; self-avoiding Gaussian polygons (Jungreis (1994)), self-avoiding equilateral polygons (Diao (1995)), etc. There are other (combinatorially and algebraically interesting) models of random knotting, such as the Petaluma model of Even-Zohar et al. (2014) or those which use elements of the braid group (e.g. in Nechaev et al. (1996)), but these are not immediately geometrically motivated like the diagram model or random space curve models.

Indeed, the primary purpose of this work is to ascertain that the conjecture holds in our model;

Theorem 1 As the number of crossings $n$ of a randomly sampled knot diagram grows large, the probability that the diagram is knotted tends to 1 exponentially quickly.

This result will follow from results on the structure of knot diagrams. However, as maps with symmetries are comparatively not well understood, we consider objects with broken symmetry: A map together with a choice of edge and a choice of direction is called rooted and considered up to the group of automorphisms which preserve the root (i.e. the directed edge) - the trivial group. We will prove results for rooted knot diagrams, which extend immediately to the unrooted case as,

$$
\left.\frac{\#\{\text { rooted } n \text {-crossing diagrams }\}}{4 n} \leq \#\{n \text {-crossing diagrams }\} \leq \# \text { rooted } n \text {-crossing diagrams }\right\} .
$$

In fact asymptotically,

$$
\frac{\#\{\text { rooted } n \text {-crossing diagrams }\}}{4 n}=\#\{n \text {-crossing diagrams }\}
$$

as a result of the following theorem:

Theorem 2 As the number of crossings $n$ of an (unrooted) knot diagram grows large, the probability that the diagram has a nontrivial automorphism group tends to 0 exponentially quickly.

These two results answer two experimentally motivated questions posed in Cantarella et al. (2015) in the affirmative. Indeed, Theorem 2 suggests that, for large $n$, experiments (c.f. Section 2.3) for unrooted knot diagrams can be run instead on rooted knot diagrams and results will only differ by a factor of $4 n$. While sampling rooted knot diagrams uniformly is still nontrivial, it is reasonably quick to generate rooted knot diagrams of 70 crossings (but nearly impossible to tabulate even all 12-crossing unrooted diagrams). 

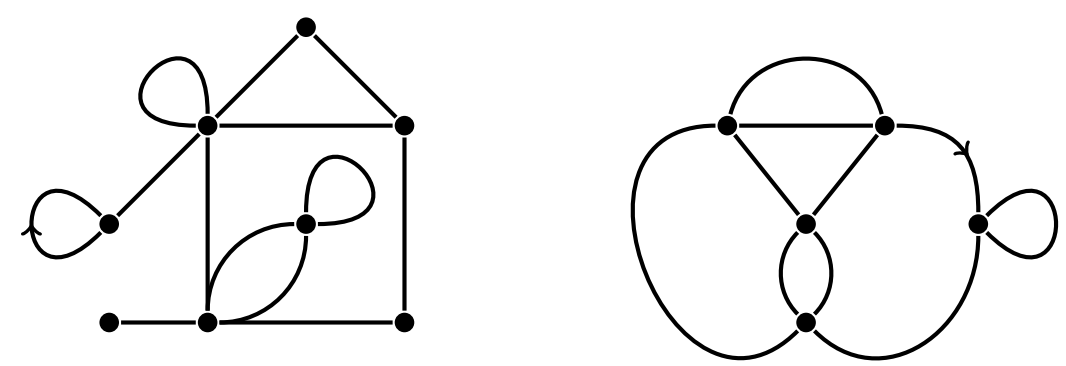

Fig. 4: A rooted planar map (left) and a rooted knot shadow (right).

We will denote by $\mathscr{K}$ the class of rooted knot diagrams, counted by the number of crossings. Unless mentioned otherwise, going onward diagrams and shadows will be assumed rooted. We note that while the counts of rooted link shadows (and hence rooted link diagrams) are known precisely (they are in bijection with a class of blossom trees), the knot condition of having precisely one knot component has made it difficult to count (or otherwise understand) $\mathscr{K}$. Indeed, there are conjectures (Schaeffer and Zinn-Justin (2004)) and computations (Coquereaux and Zuber (2015); Jacobsen and Zinn-Justin (2002); Zinn-Justin and Zuber (2009)) of the size, but there is as of yet no closed formula for the sizes $k_{n}$ of $\mathscr{K}_{n}$, the set of knot diagrams with $n$ crossings. That an innocuous condition like "only having one link component" makes counting the class of maps difficult is not unusual; indeed, for meanders as well there are still only conjectures on the asymptotics (Francesco et al. (2000)). We note that these conjectures for rooted knot shadows and meanders are both made using intuition from $2 d$ quantum gravity.

Both Theorem 1 and Theorem 2 are proved using a "pattern theorem," which states that knot diagrams almost surely contain appropriate substructure linearly often. Indeed, Sumners and Whittington's original proof of the Frisch-Wasserman-Delbrück conjecture for self-avoiding lattice polygons makes use of Kesten's Pattern Theorem for substructure in self avoiding walks. In the case of knot diagrams (or knot shadows), the appropriate substructure is that of tangle diagrams (shadows): A $2 k$-tangle shadow with $n$ crossings is a map with $n 4$-valent vertices (crossings) and one boundary vertex of degree $2 k$. A $2 k$-tangle diagram is again a tangle shadow where all (non-boundary) vertices are decorated with sign $\{+,-\}$. An example tangle diagram can be seen in Figure 6 A tangle diagram (resp. shadow) is contained in a link diagram (shadow) if there is some generic open disk on the sphere in which the diagram (shadow) is embedded that is map isomorphic (as maps on open disks) to the interior of the tangle diagram (shadow). The Pattern Theorem requires an additional hypothesis that the tangle can be attached to diagrams; essentially given a diagram $D$ in a fixed class there are sufficiently many ways to produce new diagrams in the class which contain the tangle. Although provided appropriate constructions different types of tangle can be attached, "connect summation" of 2-tangles (c.f. Figure 7 ) is sufficient to prove results on $\mathscr{K}$. The full pattern theorem for knot diagrams is then;

Theorem 3 Fix a constant $c$. Let $P$ be a tangle diagram which can be appropriately attached to knot diagrams in $\mathscr{K}$ (we will formally define attachment soon). Let $h_{n}$ be the counts of $n$-crossing diagrams in $\mathscr{K}$ who are $P$-deficient; they contain fewer than cn pairwise vertex-disjoint copies of $P$. For $0<c<1$ 


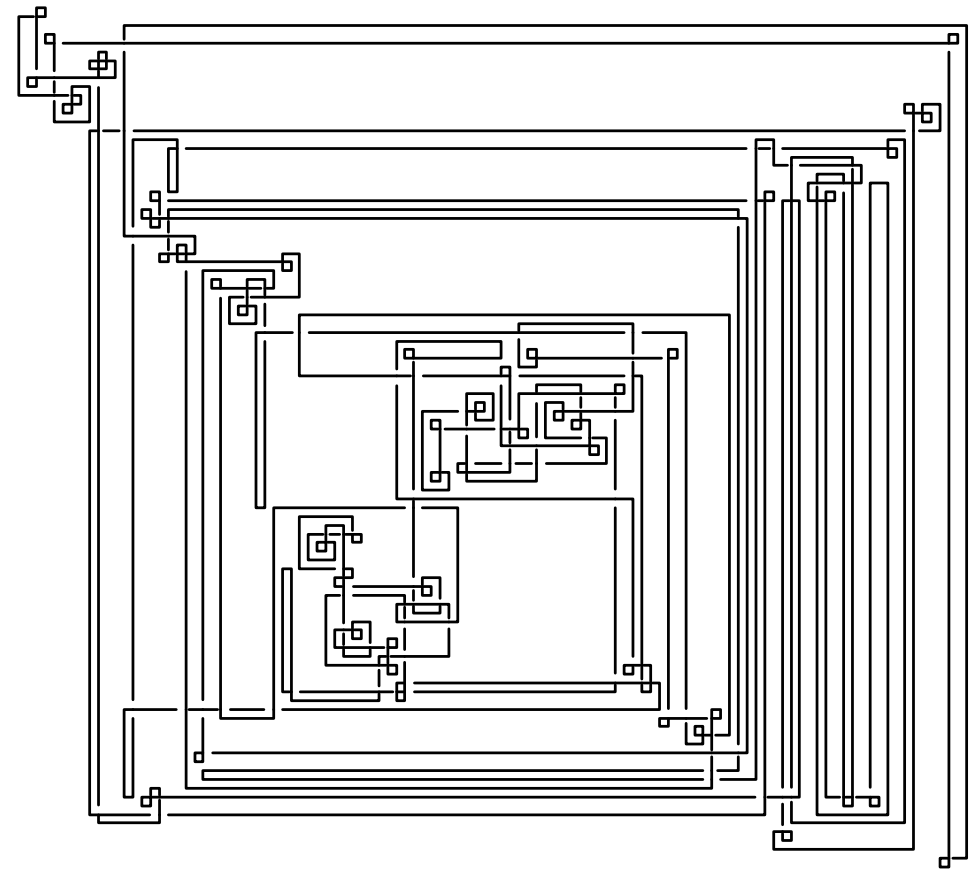

Fig. 5: A random knot diagram with 150 crossings.
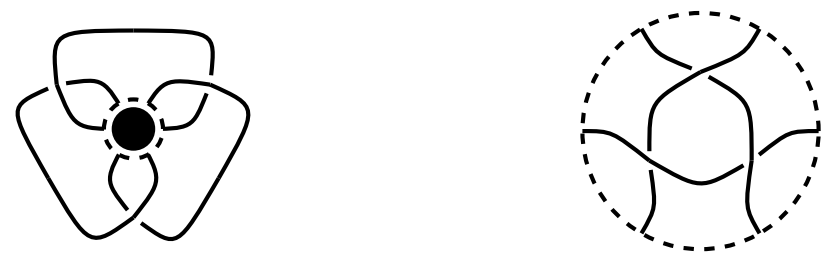

Fig. 6: A 6-tangle diagram with boundary vertex (left) and boundary vertex viewed as disk boundary (right). 
sufficiently small, there exists $0<d<1$ so that,

$$
\frac{h_{n}}{k_{n}}<d^{n}
$$

i.e. the diagrams in $\mathscr{K}$ which do not contain at least cn copies of $P$ are asymptotically exponentially rare.

As mentioned earlier, the theorem is proved for classes of rooted knot diagrams but extend immediately to unrooted knot diagrams as well. We will see by our attachment constructions that the Pattern Theorem is stronger than the bare result on unknotting (Theorem 11): Not only is every large knot diagram (rooted or unrooted) knotted, it is a highly composite knot diagram of many factors.

\section{Results}

\subsection{The Pattern Theorem}

The attachment defined in the Pattern Theorem 3 is rather technical. The Pattern Theorem itself is strongly based on results of Bender et al.(1992) and the definition of attachment is nearly the same;

Definition Let $P$ be a tangle diagram, and let $\mathscr{H}_{n}$ be the sets of knot diagrams in $\mathscr{K}_{n}$ who contain fewer than $c n$ copies of $P$ as a subtangle. The tangle diagram $P$ can be appropriately attached to knot diagrams in $\mathscr{K}$ if there exists a (possibly larger) tangle diagram $Q$ containing $P$ which can be attached to each $n$-crossing diagram $K$ in $\mathscr{H}$ in such a way that,

1. for some fixed, positive integer $k$, at least $\lfloor n / k\rfloor$ possible non-conflicting places (e.g. edge choices) of attachment exist,

2. only diagrams in $\mathscr{K}$ are produced,

3. for any diagram so produced we can identify the copies of $Q$ that have been added (i.e. we can identify the open disk which is map isomorphic to the tangle's interior) and they are all pairwise vertex-disjoint, and

4. given the copies that have been added, the original diagram and the associated places of attachment are uniquely determined.

The key piece then is the proposition,

Proposition 1 Fix a constant $c$. Let $P$ be a tangle diagram which can be appropriately attached to knot diagrams in $\mathscr{K}$. Let $\mathscr{H}$ the class of diagrams in $\mathscr{K}$ who are P-deficient. Let the generating functions for $\mathscr{K}$ and $\mathscr{H}$ be $K(z)$ and $H(z)$ respectively. For $0<c<1$ sufficiently small,

$$
r(K)<r(H),
$$

where $r(F)$ denotes the radius of convergence of $F$. By the Cauchy-Hadamard theorem, there is the equivalent result on the counting sequences;

$$
\limsup _{n \rightarrow \infty} k_{n}^{1 / n}>\limsup _{n \rightarrow \infty} h_{n}^{1 / n} .
$$


The proof of the proposition is nearly the same as that in Bender et al. (1992); it follows through without much alteration for decorated maps. Appropriate attachment constructions however require that the counts of link components remain fixed. The proposition (and hence Theorem 3 can be shown to apply to subclasses of knot diagrams, but in the case of all of $\mathscr{K}$ the construction is illustrative and quite natural; it is analogous to the connect sum operation on knots.

A 2-tangle diagram is prime if, after deleting the boundary vertex, the tangle diagram's underlying graph is at least 3 -edge connected. Let $P$ be a prime 2-tangle with at least 3 crossings and exactly one link component; let $Q$ simply be $P$. Then the following attachment, "connect sum," is admissible: Given a rooted diagram $K$ a (non-root) edge $e$, and choice of direction, the attachment of $Q$ into $K$ is given by deleting a (sufficiently small) open disk around the midpoint of $e$ and replacing it by the tangle $Q$ and smoothing edges appropriately (so there are no degree 2 vertices). It is a fact that the knot type of a connect sum of a diagram and a tangle is exactly the connect sum of the two knot types of the diagram and the tangle (once closed and viewed as a diagram). An example of this operation is given in Figure 7.

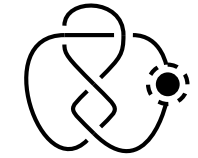

(a) Diagram with marked edge.

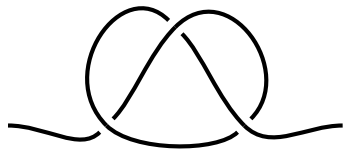

(b) A trefoil connect summand.

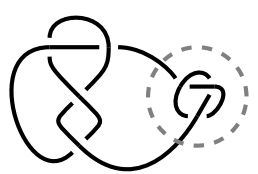

(c) After connect summation.

Fig. 7: Connect sum of a figure eight knot to a trefoil.

Then,

1. There are $2 n$ edges, so $2(2 n-1)$ locations for attachment. With $k=1$, we have that

$$
2(2 n-1) \geq\lfloor n\rfloor
$$

2. The result is indeed a diagram in $\mathscr{K}$; connect sum attachment of $Q$ does not change the number of link components (the original diagram had one, as did the tangle $Q$; the two are identified after attachment and become one link component) and the map produced is exactly 4-valent.

3. Two instances of $Q$ must be pairwise disjoint in a knot diagram as $Q$ is prime.

4. Diagram connect sum is reversible (this is covered in Cantarella et al. (2015)), and so the original diagram and places of attachment can be recovered.

Indeed, this construction together with the proposition do not entirely prove Theorem 3 In fact, the last required piece is that,

Proposition 2 The limit $\lim _{n \rightarrow \infty} k_{n}^{1 / n}$ exists and is equal to $\lim _{\sup _{n \rightarrow \infty}} k_{n}^{1 / n}$.

This proposition is of course clear in the case of link diagrams (which are counted exactly), but not immediate in the case of knot diagrams. The proof for the case of $\mathscr{K}$ is almost entirely that any two knot diagrams can be connect summed (similarly to the above attachment construction) to produce a new knot 
diagram; some care is required however as different pairs of diagrams can connect sum to produce the same knot diagram! It is hence useful to apply the framework of Bender et al.(1992) for proving so-called "smooth" growth; appropriate constructions can apply to further classes of knot diagrams as well.

Altogether, this proves Theorem 3 . We can now show the following corollary for rooted knot diagrams (which is slightly weaker than Theorem 11:

Corollary 1 Let $u_{n}$ be the number of n-crossing rooted knot diagrams which represent the unknot. Then there exists $0<d<1$ so that,

$$
\frac{u_{n}}{k_{n}}<d^{n},
$$

i.e. unknotted rooted diagrams are exponentially rare among rooted knot diagrams.

Proof: Let $P$ be the 2-tangle diagram in Figure 7(b), i.e. a prime trefoil connect summand. Unknot diagrams are certainly $P$-deficient (otherwise they would represent a knot type which has trefoil factors), hence rare by Theorem 3

\subsection{Asymmetry}

It is a theorem of Richmond and Wormald (1995) that "almost all maps are asymmetric," for maps which obey a pattern theorem, and indeed as we shall see their theorem applies to knot shadows. Notice then that almost all decorated knot shadows (e.g. knot diagrams) are asymmetric (they are arguably more asymmetric) since the decoration imposes additional constraints. Notice, for example, that the connect summation of a tangle to a diagram at a marked edge (Figure 7) can occur in 4 different ways. Say a tangle shadow $P$ is free in a class of rooted knot shadows $\mathscr{C}$ if any knot shadow obtained by removing a copy $P_{1}$ of $P$ from a shadow $K$ and re-attaching $P$ in any fashion such that the exterior legs of $P$ match up with the loose strands of $K \backslash P_{1}$ is in $\mathscr{C}$.

We restate their theorem as it will apply to knot shadows;

Theorem (Richmond and Wormald (1995)) Let $\mathscr{C}$ be a class of rooted knot shadows. Suppose that there is a tangle shadow $P$ such that for all knot shadows in $\mathscr{C}$, all copies of $P$ are pairwise disjoint and such that $P$

1. has no reflective symmetry in the plane,

2. satisfies the hypotheses for the Pattern Theorem 3 for $\mathscr{C}$, and

3. is free in $\mathscr{C}$.

Then the proportion of n-crossing shadows in $\mathscr{C}$ with nontrivial automorphisms (that need not preserve the root) is exponentially small.

Hence the proof of Theorem 2 then depends only on the appropriate construction.

Proof of Theorem 2; The 2-tangle shadow in figure 8 satisfies the hypotheses at is a prime connect summand tangle shadow and is appropriately asymmetric.

This proves then the suppositions of Schaeffer and Zinn-Justin (2004) and Coquereaux and Zuber (2015). It also follows that, asymptotically, there are simply $4 n$ times as many rooted knot shadows (or diagrams) as there are unrooted. 


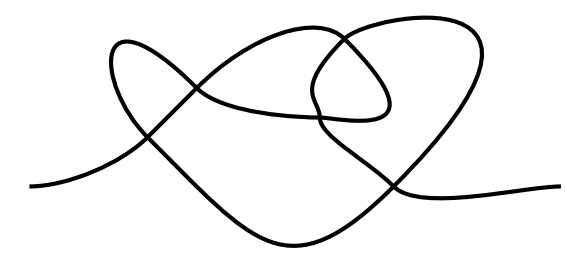

Fig. 8: A 2-tangle shadow satisfying the hypotheses for the asymmetry theorem.

\subsection{Sampling}

As we can obtain similar results for unrooted knot diagrams that we can for rooted knot diagrams, we can use the bijection of Schaeffer (1997) between rooted 4-valent maps and blossom trees to sample knot shadows efficiently. This yields the following uniform sampling pipeline, which while imperfect, yields results for sizes ten times the tabulation strategy of Cantarella et al. (2015):

1. Sample a rooted 4-valent planar map with $n$ vertices (e.g., using PlanarMap of Schaeffer (1999)). This can be done quickly, as the blossom trees are nearly the same as binary trees.

2. Reject until the map is a rooted knot shadow (i.e. it has one link component). This becomes exponentially unlikely to succeed (because of the Pattern Theorem for link shadows).

3. For each of the $n$ crossings of the shadow, sample a sign from $\{+,-\}$, yielding a rooted knot diagram. This is trivial.

An example of a random knot diagram produced as such appears in Figure 5. Notice that the embedding of the graphic is independent of the actual knot diagram object.

The following experiment produced the data of Figure 9. For each $3 \leq n \leq 70$, sample 20000 knot diagrams using the above procedure, skipping a sample if it does not produce a knot shadow after 150 attempts (this number is chosen arbitrarily so that the experiment could complete in a reasonable amount of time). Classify the knot type (ignoring chirality) by calculating the HOMFLY-PT polynomial of the diagram. The experiment took 3 hours to complete on an Intel Core2 Duo $2.5 \mathrm{Ghz}$ laptop, while it took nearly 7 days with an Amazon Cloud Messaging set up (working in parallel on 20 or so machines) to classify precisely all of the 1.6 billion 10-crossing diagrams in Cantarella et al. (2015). We note that indeed we take fewer samples in this present experiment, but that it is regardless required of the tabulation scheme that every diagram be tabulated (or else the sampling measure would not be uniform). The power is hence that we need not count the entire space (of rooted knot diagrams) to estimate results therein.

Notably, the data shows (as expected) that the unknot ratio (indeed, the ratio of all fixed knot types observed) tends to zero exponentially quickly. Furthermore, there is a point at around 40 crossings where the ratio of trefoils surpasses that of unknots. Indeed, we show in the full version Chapman (2016) that no one knot type is most common for all time. 

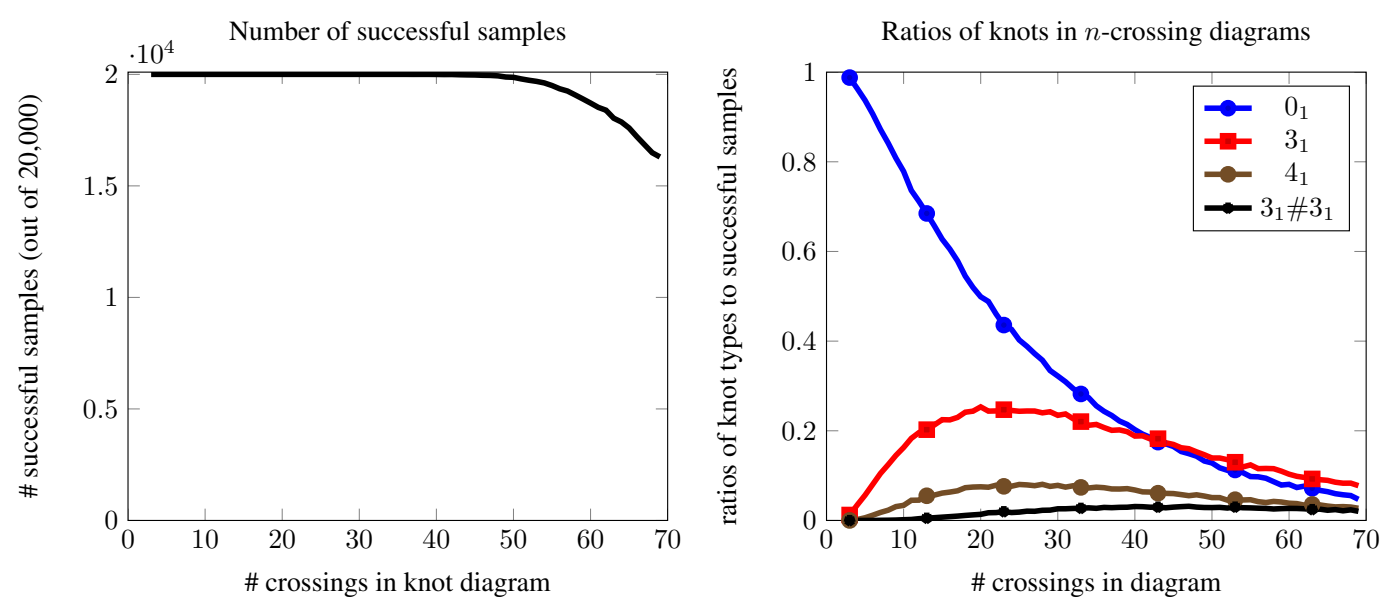

Fig. 9: Number of successful samples (left). Plot of various knot types recorded during sampling (right).

\section{Conclusion}

We have covered some of the results for general knot shadows and knot diagrams. However, the machinery permits more (provided appropriate constructions). For example, we provide a proof in the full version (Chapman (2016)) of the Pattern Theorem (and beyond) for subclasses of knot diagrams; reduced diagrams, who are at least 2-vertex connected, and prime diagrams, who are at least 4-edge connected. Respectively these two subclasses refer to diagrams without "nugatory crossings" whose crossing sign does not affect knot type and diagrams who cannot be realized as a diagram connect sum of two smaller diagrams, and are interesting topologically.

As well, the topological map language enables one to prove further results for different kinds of knot diagrams. For example, by instead considering knot shadows on orientable surfaces of arbitrary genus (rather than simply the sphere), one arrives at the class of virtual knot diagrams of Kauffman (1999). We note that Gauss diagrams describe knot diagrams of arbitrary genus, while planar decorated planar link diagrams describe spherical diagrams of arbitrarily many link components. Trying to restrict either to spherical knot diagrams is difficult.

As the Frisch-Wasserman-Delbrück conjecture holds for the random diagram model, it is hoped that the powerful tools that exist for maps on surfaces can be applied to prove further results for the random diagram model which are physically relevant.

\section{Acknowledgements}

The author is extremely grateful to his advisor Jason Cantarella, for his support, advice, and for introducing him to the knot tabulation project (alongside Matt Mastin) and suggesting he prove Theorem 1 . The author is also grateful to the summer school on applied combinatorics at the University of Saskatchewan and CanaDAM thereafter. The author also is indebted to funding from the NSF (grant DMS-1344994 of the RTG in Algebra, Algebraic Geometry, and Number Theory, at the University of Georgia), PIMS, the Simons Center, and the AMS through which he was able to introduce his work to others. The author is 
grateful for conversations with Julien Courtiel, Elizabeth Denne, Chris Duffy, Chaim Even-Zohar, Éric Fusy, Gary Iliev, Rafał Komendarczyk, Neal Madras, Kenneth Millett, Marni Mishna, Erik Panzer, Jason Parsley, Eric Rawdon, Andrew Rechnitzer, Clayton Shonkwiler, Chris Soteros, Karen Yeats, and many others. Lastly, the author is grateful to Jean Gutt for his assistance in translating the abstract into French.

\section{References}

V. I. Arnol'd. The geometry of spherical curves and the algebra of quaternions. Russian Mathematical Surveys, 50(1):1, 1995. URL http://stacks.iop.org/0036-0279/50/i=1/a=R01.

E. A. Bender, Z.-C. Gao, and L. Richmond. Submaps of maps. I. General 0-1 laws. Journal of Combinatorial Theory, Series B, 55(1):104 - 117, 1992. ISSN 0095-8956. doi: http://dx.doi. org/10.1016/0095-8956(92)90034-U. URL http://www.sciencedirect.com/science/ article/pii/009589569290034U.

J. Cantarella, H. Chapman, and M. Mastin. Knot probabilities in random diagrams. arXiv preprints, Dec. 2015.

H. Chapman. Asymptotic laws for random knot diagrams. in preparation, 2016.

R. Coquereaux and J.-B. Zuber. Maps, immersions and permutations. ArXiv e-prints, July 2015.

M. Delbrück. Knotting Problems in Biology, chapter 5, pages 55-63. American Mathematical Society, 1962. doi: 10.1090/psapm/014. URL http://dx.doi.org/10.1090/psapm/014.

Y. Diao. The knotting of equilateral polygons in $\mathbb{R}^{3}$. Journal of Knot Theory and Its Ramifications, 04(02): 189-196, 1995. doi: 10.1142/S0218216595000090. URL http://www.worldscientific. $\mathrm{com} / \mathrm{doi} / \mathrm{abs} / 10.1142 / \mathrm{s} 0218216595000090$.

Y. Diao, C. Ernst, and U. Ziegler. Generating large random knot projections. In Physical and Numerical Models in Knot Theory, chapter 23, pages 473-494. World Scientific, 2012. doi: 10.1142/ 9789812703460_0023. URL http://www.worldscientific.com/doi/abs/10.1142/ $9789812703460 \_0023$

N. Dunfield, A. Hirani, M. Obeidin, A. Ehrenberg, S. Bhattacharyya, and D. Lei. Random Knots: A preliminary report, 2014. URL http://www.math.uiuc.edu/ nmd/preprints/slides/ random_knots.pdf.

C. Even-Zohar, J. Hass, N. Linial, and T. Nowik. Invariants of random knots and links. Dec 2014. URL http://arxiv.org/abs/1411.3308v2.

P. D. Francesco, O. Golinelli, and E. Guitter. Meanders: exact asymptotics. Nuclear Physics B, 570 (3):699 - 712, 2000. ISSN 0550-3213. doi: http://dx.doi.org/10.1016/S0550-3213(99)00753-1. URL http://www.sciencedirect.com/science/article/pii/s0550321399007531

H. L. Frisch and E. Wasserman. Chemical topology 1. J. Am. Chem. Soc., 83(18):3789-3795, sep 1961. doi: 10.1021/ja01479a015. URL http://dx.doi.org/10.1021/ja01479a015 
J. Jacobsen and P. Zinn-Justin. A transfer matrix approach to the enumeration of knots. J. Knot Theory Ramifications, 11(5):739-758, 2002. ISSN 0218-2165.

D. Jungreis. Gaussian random polygons are globally knotted. Journal Of Knot Theory And Its Ramifications, 03(04):455-464, 1994. doi: 10.1142/S0218216594000332. URL http://www . worldscientific.com/doi/abs/10.1142/s0218216594000332.

L. H. Kauffman. Virtual knot theory. European Journal of Combinatorics, 20(7):663 - 691, 1999. ISSN 0195-6698. doi: http://dx.doi.org/10.1006/eujc.1999.0314. URL/http://www . sciencedirect. com/science/article/pii/s0195669899903141.

S. K. Nechaev, A. Y. Grosberg, and A. M. Vershik. Random walks on braid groups: Brownian bridges, complexity and statistics. Journal of Physics A: Mathematical and General, 29(10):2411-2433, 1996.

L. Richmond and N. Wormald. Almost all maps are asymmetric. Journal of Combinatorial Theory, Series B, 63(1):1 - 7, 1995. ISSN 0095-8956. doi: http://dx.doi.org/10.1006/jctb.1995.1001. URL http://www.sciencedirect.com/science/article/pii/s0095895685710015.

G. Schaeffer. Bijective census and random generation of eulerian planar maps with prescribed vertex degrees. The Electronic Journal of Combinatorics, 4(1):Research paper R20, 14 p.-Research paper R20, 14 p., 1997. URL http://eudml.org/doc/119255.

G. Schaeffer. Random sampling of large planar maps and convex polyhedra. In Proceedings of the Thirtyfirst Annual ACM Symposium on Theory of Computing, STOC '99, pages 760-769, New York, NY, USA, 1999. ACM. ISBN 1-58113-067-8. doi: 10.1145/301250.301448. URL http://doi .acm. org/10.1145/301250.301448.

G. Schaeffer and P. Zinn-Justin. On the asymptotic number of plane curves and alternating knots. Experiment. Math., 13(4):483-493, 2004. ISSN 1058-6458.

D. W. Sumners and S. G. Whittington. Knots in self-avoiding walks. J. Phys. A: Math. Gen., 21(7): 1689-1694, apr 1988. doi: 10.1088/0305-4470/21/7/030. URL http://dx.doi.org/10.1088/ $0305-4470 / 21 / 7 / 030$.

P. Zinn-Justin and J.-B. Zuber. Knot theory and matrix integrals. In Random Matrix Theory. 2009. Eds Akemann, Baik and Di Francesco. 